\title{
Preservation of Seed Crystals in Feedstock Melting for Cast Quasi-Single Crystalline Silicon Ingots
}

\author{
Zaoyang Li, ${ }^{1,2}$ Lijun Liu, ${ }^{1}$ Yunfeng Zhang, ${ }^{3}$ Qingchao Meng, \\ Zhiyan $\mathrm{Hu}^{3}{ }^{3}$ and Genshu Zhou ${ }^{2}$ \\ ${ }^{1}$ Key Laboratory of Thermo-Fluid Science and Engineering, Ministry of Education, School of Energy and Power Engineering, \\ Xian Jiaotong University, Xian, Shaanxi 710049, China \\ ${ }^{2}$ State Key Laboratory for Mechanical Behavior of Materials, School of Materials Science and Engineering, Xian Jiaotong University, \\ Xian 710049, China \\ ${ }^{3}$ Yingli Green Energy Holding Co., Ltd., Baoding, Hebei 071051, China
}

Correspondence should be addressed to Lijun Liu; ljliu@mail.xjtu.edu.cn

Received 29 May 2013; Accepted 12 September 2013

Academic Editor: Chun-Sheng Jiang

Copyright ( 2013 Zaoyang Li et al. This is an open access article distributed under the Creative Commons Attribution License, which permits unrestricted use, distribution, and reproduction in any medium, provided the original work is properly cited.

The preservation of seed crystals is important for the casting of quasi-single crystalline (QSC) silicon ingots. We carried out transient global simulations of the feedstock melting process in an industrial-sized directional solidification (DS) furnace to investigate key factors influencing seed preservation. The power distribution between the top and side heaters is adjusted in the conventional furnace for multicrystalline silicon ingots and in the evolved furnace with a partition block for QSC silicon ingots. The evolution of the solid-liquid interface for melting and the temperature distribution in the furnace core area are analyzed. The power distribution can influence the temperature gradient in the silicon domain significantly. However, its effect on seed preservation is limited in both furnaces. Seed crystals can be preserved in the evolved furnace, as the partition block reduces the radiant heat flux from the insulation walls to the heat exchange block and prevents the heat flowing upwards under the crucible. Therefore, the key to seed preservation is to control radiant heat transfer in the DS furnace and guarantee downward heat flux under the crucible.

\section{Introduction}

Quasi-single crystalline (QSC) silicon ingots have been popular in photovoltaic applications recently because of their lower manufacturing cost, higher throughput, and higher conversion efficiency for solar cells [1]. The QSC silicon ingot is cast by directional solidification (DS) technology evolved from the conventional DS method for multicrystalline silicon (mc-Si) ingots [2]. To induce single crystal growth, seed crystals approximately $20 \mathrm{~mm}$ thick are packed closely at the bottom of the crucible. Solar grade silicon feedstock is then loaded into the crucible and melted. After melting, liquid silicon begins to crystallize onto the seeds in the same crystallographic orientation and the seed crystals must therefore be preserved during the melting process. In addition, thin seed crystals, which are preferable to reduce costs, are more likely to be melted. Therefore, it is necessary to investigate factors that could affect seed preservation during feedstock melting for growing single crystals and reducing costs.

Some research has been carried out to study the phenomena and mechanisms in the casting of QSC silicon ingots. Ma et al. [1], Yu et al. [3], Black et al. [4], and Gao et al. [5] numerically investigated heat and mass transport in the crystal growth process. They provided advice for controlling the temperature distribution, growth rate, solid-liquid interface shape, and impurity transport and for improving ingot quality. Gu et al. [6] and Stoddard et al. [7] introduced basic characteristics of the QSC silicon ingots and performance of the QSC silicon solar cells. Witting et al. [8], Tachibana et al. [9], and Tsuchiya et al. [10] tried to establish the mechanisms of defect generation in the QSC silicon ingots. These studies are helpful for understanding the casting process and improving the quality of QSC silicon ingots. However, little research has been conducted on seed 
crystal preservation. As the seed crystals lie on the bottom of the crucible and come into close contact with the feedstock, special attention should be given to seed preservation during the melting process.

Heat transfer is the most important factor that determines melting of the silicon feedstock. During melting, heat is transferred from the hot heaters to the feedstock. A closed insulation chamber is fixed outside the heaters to decrease heat loss. To preserve the seed crystals located at the bottom of the crucible, the feedstock should be melted from top to bottom. Therefore, adjusting the power distribution among different heaters in a multi-heater DS furnace is one possible way of changing the heat transfer direction and influencing the melting sequence [11]. Adding a partition block in the furnace chamber is another way of changing the pathway for radiative heat transfer and controlling the melting process. In this study, we numerically investigate the effects of power distribution and partition block design on seed preservation in the melting process.

\section{Model Description}

Though the QSC silicon ingot is cast by evolved DS technology, our investigation begins from the conventional DS furnace for the mc-Si ingots and establishes whether the seed crystals can be preserved in such a furnace. A schematic of the conventional furnace for $450 \mathrm{~kg}$ ingots is shown on the left in Figure 1. An insulation partition block (no. 13) is installed in the evolved furnace to change the radiation pathway, as shown on the right in Figure 1. Both the DS systems consist of quartz crucible, graphite susceptor, heat exchange block, graphite resistance heaters, insulations, and chamber walls. The crucible volume is $84 \times 84 \times 40 \mathrm{~cm}^{3}$ and the ingot height is $25 \mathrm{~cm}$. A thermocouple (TC) is installed at the upper center of the heat exchange block to monitor the temperature at the crucible bottom. The top and side heaters in the furnaces allow for adjustment of the power distribution between the two heaters. During the melting process, the insulation is closed and heat is transferred from the hot heaters to the feedstock mainly by radiation. For casting the mc-Si ingot, the silicon feedstock can be melted from any direction. For the QSC silicon ingot, the top feedstock should be melted first and the seed crystals on the crucible bottom must be preserved during the melting process.

To describe the melting process accurately, transient global simulations including thermal conduction, thermal radiation, melt convection, gas flow, and phase change were carried out. The algorithms for global modeling of heat transfer in a DS furnace have been published elsewhere [12, 13]. The solid-liquid interface may be highly irregular during melting. Therefore, we use the enthalpy method on fixed grids to capture the interface [14]. It is assumed that the feedstock density is equal to that of the solidified ingot. The energy equation governing heat transfer in the silicon domain is

$$
\begin{aligned}
\frac{\partial(\rho h)}{\partial t}+\nabla \cdot(\rho h \vec{u})= & \nabla \cdot(\lambda \nabla T)+\frac{\partial(\rho \Delta H)}{\partial t} \\
& +\nabla \cdot(\rho \Delta H \vec{u}),
\end{aligned}
$$

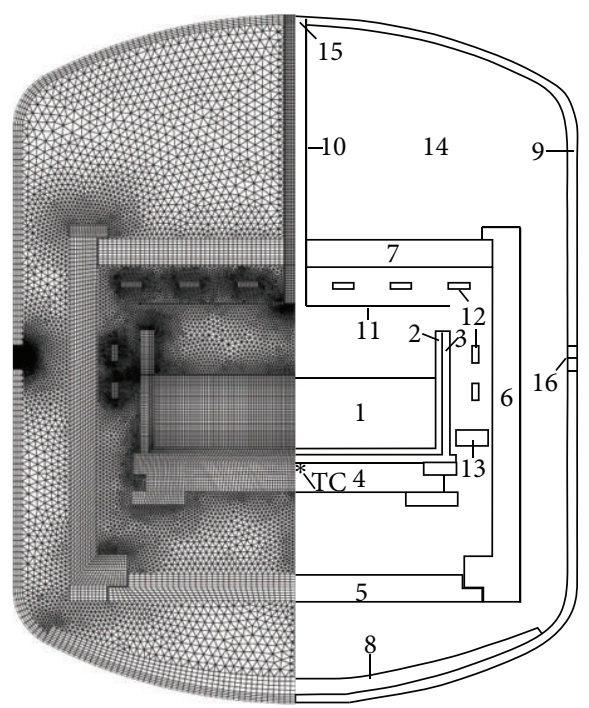
(1) Silicon domain
(10) Gas tube
(2) Crucible
(11) Cover
(3) Susceptor
(12) Heaters
(4) Heat exchange block
(5-7) Insulations
(13) Partition block
(8) Insulated pad
(14) Gas domain
(9) Wall
(15) Gas inlet
(16) Gas outlet

FIGURE 1: Configurations and computational grids of two industrialsize DS furnaces.

where $\rho, h, t, \vec{u}, \lambda$, and $T$ are the density, sensible enthalpy, time, velocity vector, heat conductivity, and temperature, respectively. $\Delta H$ is the latent heat for phase changes and is defined as

$$
\Delta H= \begin{cases}L, & T>T_{m} \\ 0<\Delta H<L, & T=T_{m} \\ 0, & T<T_{m},\end{cases}
$$

where $L$ is the latent heat of the phase change and $T_{m}$ is the silicon melting point.

The above numerical model is first validated by comparison with experimental data. Then, the power distribution ratio between the top and side heaters is set to $5: 5$ and $10: 0$ in both furnaces to investigate the effect of power distribution and partition block on seed preservation. The ratio of $5: 5$ is adopted in the experiment. The $10: 0$ ratio in the simulations means that the top heater occupies all power and heat transfer is enhanced from the top to the bottom of the silicon domain, which is beneficial to seed preservation. The total power applied in the melting process is the same as that in the experiment and is maintained the same for all cases.

\section{Results and Discussion}

3.1. Validation of Numerical Model. We use the enthalpy method and calculation of global heat transfer to simulate the melting process. Figure 2 (a) shows the simulated temperature profile at the top surface of the silicon feedstock. There are 


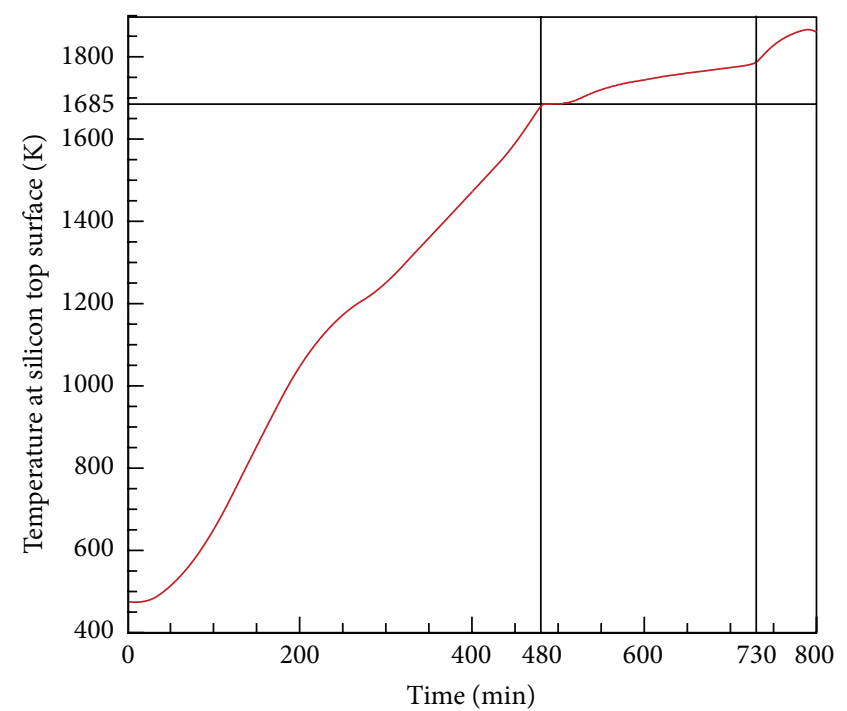

- Calculation

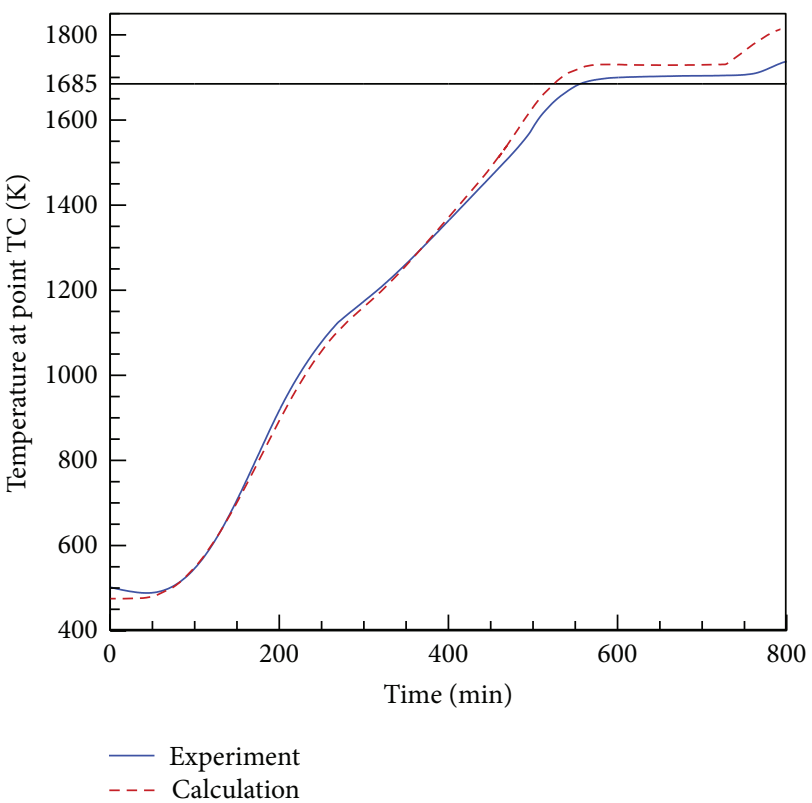

(b)

Figure 2: Temperature evolution at (a) silicon top surface and (b) point TC in the conventional DS furnace for a power distribution ratio of $5: 5$.

two stages in the melting process: heating of the furnace and melting of the feedstock. The heating stage lasts from 0 to $480 \mathrm{~min}$ and the furnace temperature increases quickly. The temperature at the silicon top surface reaches the melting point of $1685 \mathrm{~K}$ at $480 \mathrm{~min}$ and the feedstock begins to melt from the top. The temperature then increases relatively slowly from 480 to $730 \mathrm{~min}$, as the feedstock absorbs heat during melting. There exists a turning point at $730 \mathrm{~min}$ when the temperature begins to increase rapidly after complete melting. Evolution of the temperature in Figure 2(a) presents a reasonable melting process for the $\mathrm{mc}-\mathrm{Si}$ ingot.

To validate the numerical model, we compared the simulations with experimental data at a power distribution ratio of $5: 5$ in the conventional furnace. Figure 2(b) shows the experimental and simulated temperature evolutions at point TC. As shown in Figure 2(b), the maximal difference in the temperature at point TC is less than $40 \mathrm{~K}$ during the melting process between the prediction and the experiment data. The relative deviation is less than $2.4 \%$. Furthermore, this discrepancy does not change the trend of the prediction compared with the experiment. Both results show that the temperature at point TC is greater than the silicon melting point of $1685 \mathrm{~K}$ after about $550 \mathrm{~min}$ in the melting process, indicating that the temperature below the crucible is higher than that in the feedstock and the seed crystals are likely to be melted. Considering the high complexity of heat transfer in the furnace and the uncertainty of the temperaturedependent physical properties of materials at high temperature, the agreement between the numerical predictions and the experiment is quite satisfactory and the numerical model can reveal the essential phenomena of heat transfer in the melting process.
3.2. Modeling of Melting Process in the Conventional Furnace. After validating the numerical model for the melting process, we investigated the melting sequence of the feedstock in the crucible. As shown in Figure 2(a), the time spent on melting the feedstock is approximately $4 \mathrm{~h}$, so we chose five time points to analyze the entire melting process at 480, 540, 600, 660, and $720 \mathrm{~min}$. Evolutions of the solid-liquid interface at these times are shown in Figure 3. From Figure 3(a), we see that the feedstock begins to melt from the top corner where it is in contact with the crucible wall. The temperature on the crucible wall is usually high as it is close to the side heater, as shown in Figure 1. After the initial stage, the top layer of feedstock begins to melt from top to bottom, as shown in Figures 3(b) to 3(c). If the seed crystals are laid on the crucible bottom, they could be preserved with this trend going on to the final stage. However, the side feedstock near the crucible wall is melted in Figure 3(b) and further in Figure 3(c). The seed crystals begin to melt in Figure 3(c) and are completely melted in Figures 3(d) and 3(e). Therefore, the seed crystals are not preserved in the conventional furnace with a power distribution ratio of $5: 5$.

To avoid heat transferring from the side heater into the crucible, we adjusted the power distribution between the top and side heaters to $10: 0$. This method could change the heat transfer direction significantly and is likely to affect the melting sequence of the feedstock. Figure 4 presents the evolutions of the solid-liquid interface for the power distribution ratio of $10: 0$ at the same time intervals as those in Figure 3. Because of the reduction in side heating power, melting of the side feedstock near the crucible in Figure 3(b) disappears in Figure 4(b). The seed crystals located at the crucible bottom still exist in Figure 4(c), which 


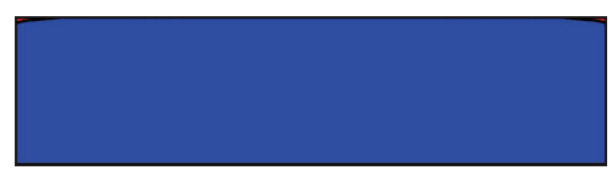

(a)

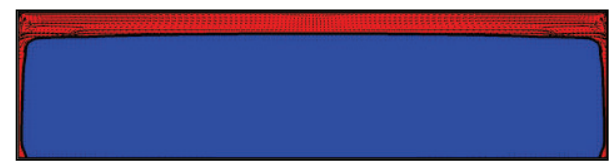

(b)

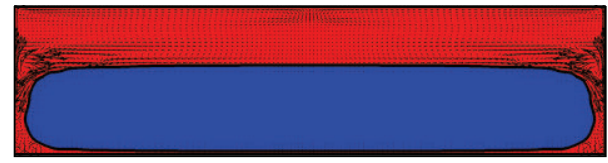

(c)

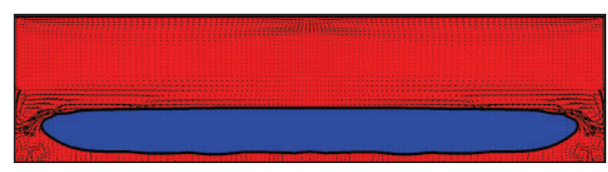

(d)

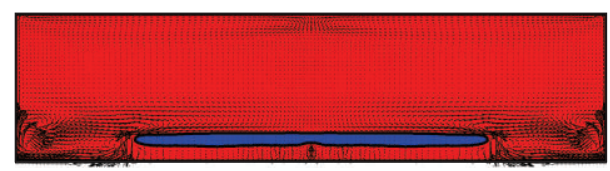

(e)

FIgURE 3: Melting process in conventional DS furnace for power distribution ratio of 5:5: (a) $480 \mathrm{~min}$; (b) $540 \mathrm{~min}$; (c) $600 \mathrm{~min}$; (d) $660 \mathrm{~min}$; (e) $720 \mathrm{~min}$.

is different from that in Figure 3(c). However, the bottom of the seed crystals is melted in Figures 4(d) to 4(e), though the height of the melted layer is less than that in Figures 3(d) and 4(e). Therefore, adjusting the power distribution in the conventional furnace does not guarantee preservation of the seed crystals.

To explain why the seed crystals melt in the conventional furnace, especially when the top heater occupies all the power, the temperature distributions inside the feedstock, quartz crucible, susceptor, and heat exchange block just before melting are shown in Figure 5. Figures 5(a) and 5(b) show the temperature distributions for the power distribution ratio of $5: 5$ and $10: 0$, respectively. The temperature at the feedstock top in Figure 5(b) is higher than that in Figure 5(a), while the temperature at the bottom is lower. These results are reasonable as the top heater occupies more power in Figure 5(b) and more heat is transferred from top to bottom. The isotherms in the lower part of the crucible side wall indicate that heat transfers into the silicon feedstock in both cases. This occurs mainly because of the reflection of radiant heat from the hot side insulation to the crucible outside wall. Therefore, adjusting the power distribution cannot prevent melting of the feedstock near the crucible side wall. It should also be noted from the temperature distribution that heat is transferred from the bottom of the heat exchange block

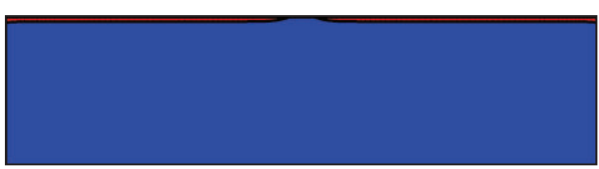

(a)

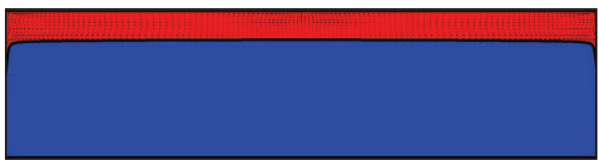

(b)

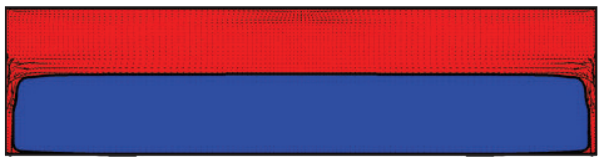

(c)

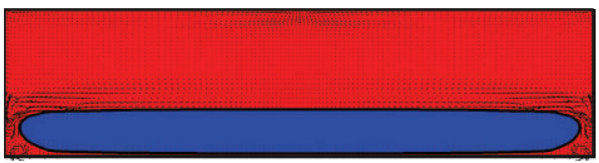

(d)

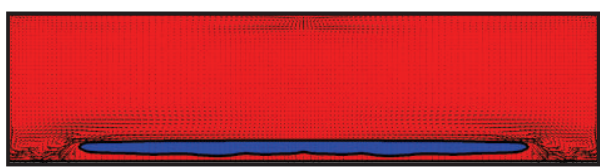

(e)

FIGURE 4: Melting process in conventional DS furnace for power distribution ratio of 10 : 0: (a) $480 \mathrm{~min}$; (b) $540 \mathrm{~min}$; (c) $600 \mathrm{~min}$; (d) $660 \mathrm{~min}$; (e) $720 \mathrm{~min}$.

to its top. The reverse heat flux originates mainly from the reflection of radiant heat at the insulation walls and could melt the seed crystals located at the crucible bottom. Based on the above analysis, we conclude that adjusting the power distribution ratio to $10: 0$ could reduce the heat reflected to the heat exchange block slightly. However, reverse heat flux cannot be avoided, and it is difficult to preserve the seed crystals in the conventional furnace.

3.3. Seed Preservation during Melting of Feedstock in the Evolved Furnace. The above analysis shows that the reverse heat flux in the heat exchange block caused by reflection is harmful to seed preservation in the conventional furnace. Therefore, we installed a partition block in the space just above the heat exchange block and between the crucible and side insulation, as shown on the right in Figure 1. The design could interrupt the heat radiated from the side insulation to the heat exchange block. Then we investigated the effect of partition block on radiative heat transfer and seed preservation in the evolved furnace. Figure 6 presents isotherms in the core area of the evolved furnace for two cases with different power distribution. It is exciting that the heat in the heat exchange block is transferred from top to bottom even when the side heater shares power. The partition block could reduce the reflected radiant heat to the side and 


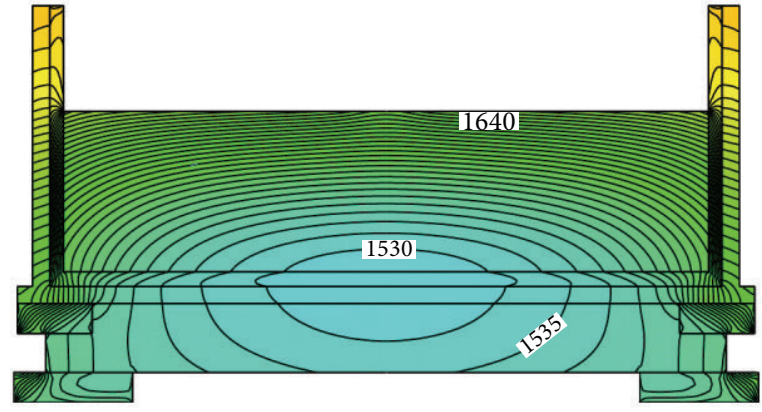

(a)

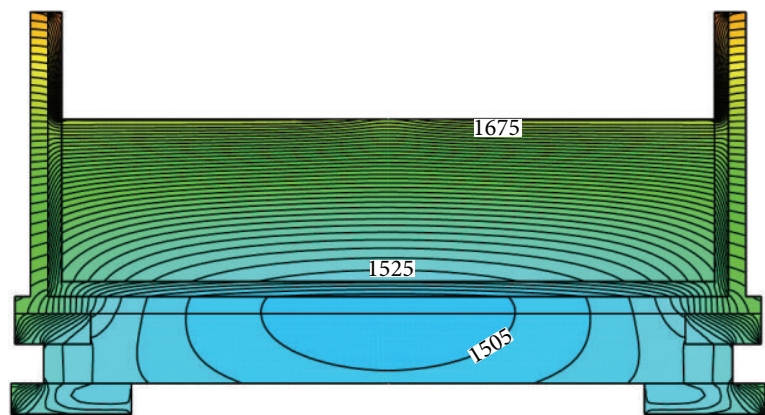

(b)

FIGURE 5: Temperature distribution ( $5 \mathrm{~K}$ between two isotherms) before melting of feedstock in conventional DS furnace for different power distribution ratios: (a) $5: 5$; (b) $10: 0$.

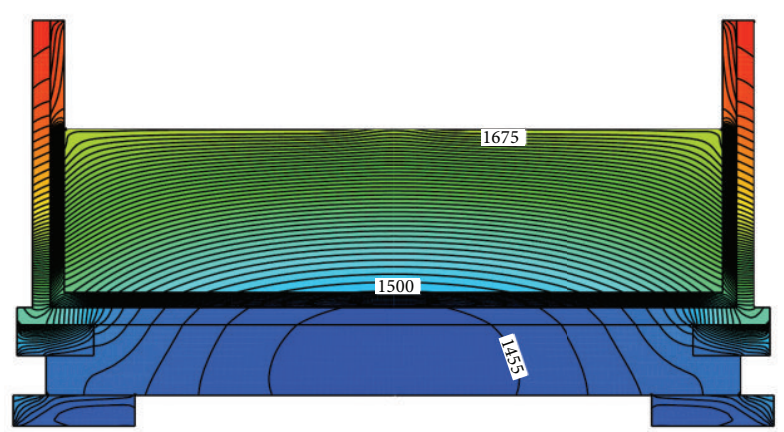

(a)

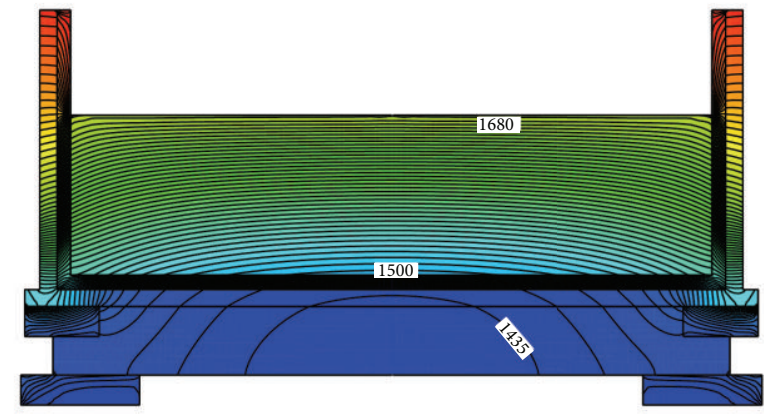

(b)

FIGURE 6: Temperature distribution ( $5 \mathrm{~K}$ between two isotherms) before melting of feedstock in evolved DS furnace for different power distribution ratios: (a) $5: 5$; (b) $10: 0$.

bottom walls of the heat exchange block significantly. The seed crystals are likely to be preserved in the melting process.

Figure 7 shows the evolution of the solid-liquid interface in the evolved furnace for a power distribution of $5: 5$. Melting of the feedstock starts from the top corner as for the conventional furnace. After the initial stage, the feedstock is melted from top to bottom and this trend continues to the end as shown in Figures 7(a) to 7(e). Seed crystals are preserved throughout the process. However, the feedstock near the crucible side wall begins melt in Figure 7(b), and it is completely melted in Figure 7(c). The seed crystals near the crucible side wall also melt as shown in Figure 7(e).

To reduce the number of melted seed crystals near the crucible, we adjusted the power distribution ratio to $10: 0$. Figure 8 presents evolutions of the solid-liquid interface in the evolved furnace for power distribution ratio of $10: 0$. The feedstock near the crucible side wall is still melted, but not as severely as in Figure 7. The preserved seed crystals in Figure 8(e) are slightly larger than those in Figure 7(e) because of the change in power distribution. Therefore, the partition block design can preserve the seed crystals, while the effect of power distribution is limited.

Based on the above analysis, a partition block was installed in an experimental DS furnace to produce QSC silicon ingots with the top heater occupying all the heating power. Figure 9 shows the longitudinal section of a silicon brick cut from the ingot edge. The width and height of the brick are 156 and $250 \mathrm{~mm}$, respectively. The polycrystalline grains originate from the crucible side wall and propagate towards the center and top of the ingot. The single crystal growth is induced by the unmelted seed crystals at the crucible bottom. The seed crystals near the crucible side wall are melted and the seed-crystal interface is slightly convex, both of which are consistent with the case predicted in Figure $8(\mathrm{e})$. By carefully controlling the melting process, the height of the preserved seed crystals is approximately $10 \mathrm{~mm}$ in the central part.

\section{Conclusions}

We numerically simulated the melting of feedstock in the industrial DS process and tried to determine which factors influence seed crystal preservation for casting QSC silicon ingots. The evolution of solid-liquid interfaces when melting and the temperature distribution in the furnace are compared for different power distributions and furnace designs. Investigations show that raising the top heating power can increase 


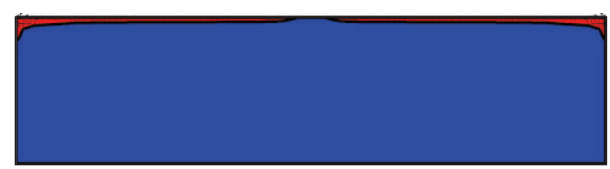

(a)

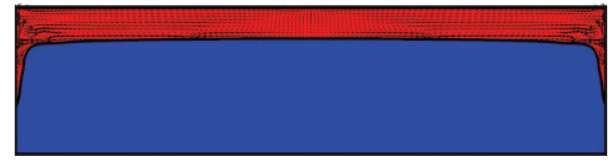

(b)

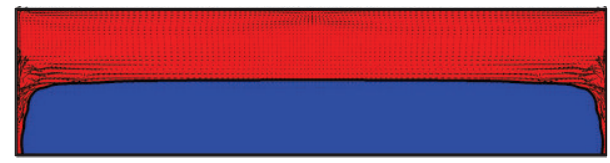

(c)

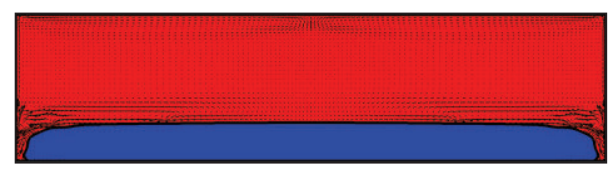

(d)

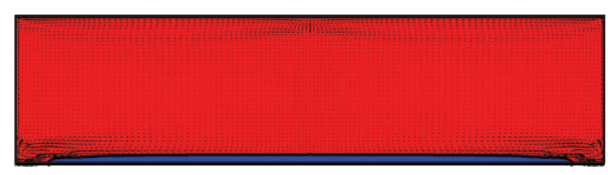

(e)

FIGURE 7: Melting process in evolved DS furnace for power distribution ratio of 5:5: (a) $480 \mathrm{~min}$; (b) $540 \mathrm{~min}$; (c) $600 \mathrm{~min}$; (d) $660 \mathrm{~min}$; (e) $720 \mathrm{~min}$.

the temperature gradient and enhance the downward heat flux in the silicon domain significantly. However, the seed crystals cannot be preserved even though the top heater occupies all the power in the conventional furnace for the mc$\mathrm{Si}$ ingots. The reflection of radiant heat from the insulation walls to the heat exchange block, which leads to an upward heat flow under the crucible, is responsible for the melting of the seed crystals. Addition of a partition block in the evolved furnace for the QSC silicon ingots can reduce the reflected heat from insulation to heat exchange block, guarantee the downward heat flow under the crucible, and preserve the seed crystals. Raising the top heating power in the evolved furnace is also beneficial for seed preservation, but its effect is limited. The numerical simulations indicate that the most important factor for seed preservation in the casting of QSC silicon ingots is to control the radiant heat transfer in the DS furnace and guarantee downward heat flux under the crucible.

\section{Acknowledgments}

This research was supported by the National Natural Science Foundation of China (no. 51176148), the Fundamental Research Funds for the Central Universities of China

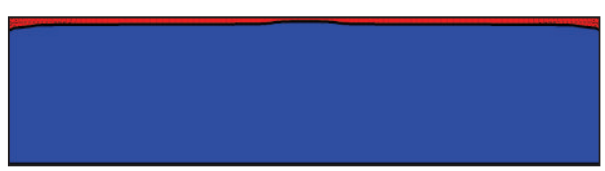

(a)

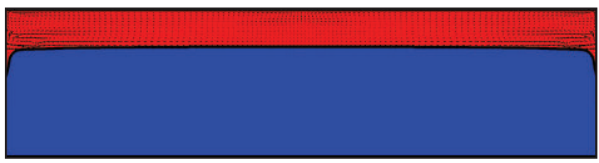

(b)

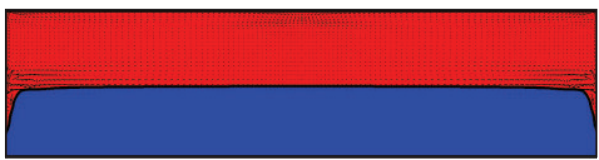

(c)

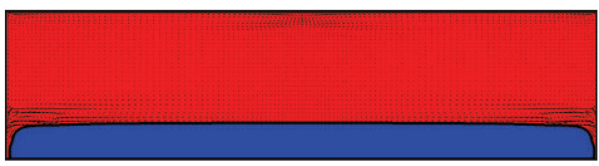

(d)

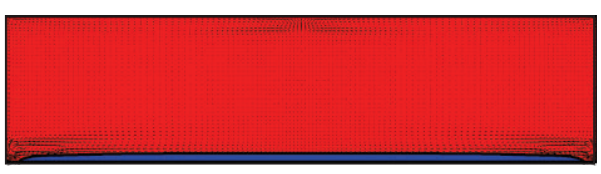

(e)

FIGURE 8: Melting process in evolved DS furnace for power distribution ratio of 10:0: (a) $480 \mathrm{~min}$; (b) $540 \mathrm{~min}$; (c) $600 \mathrm{~min}$; (d) $660 \mathrm{~min}$; (e) $720 \mathrm{~min}$.

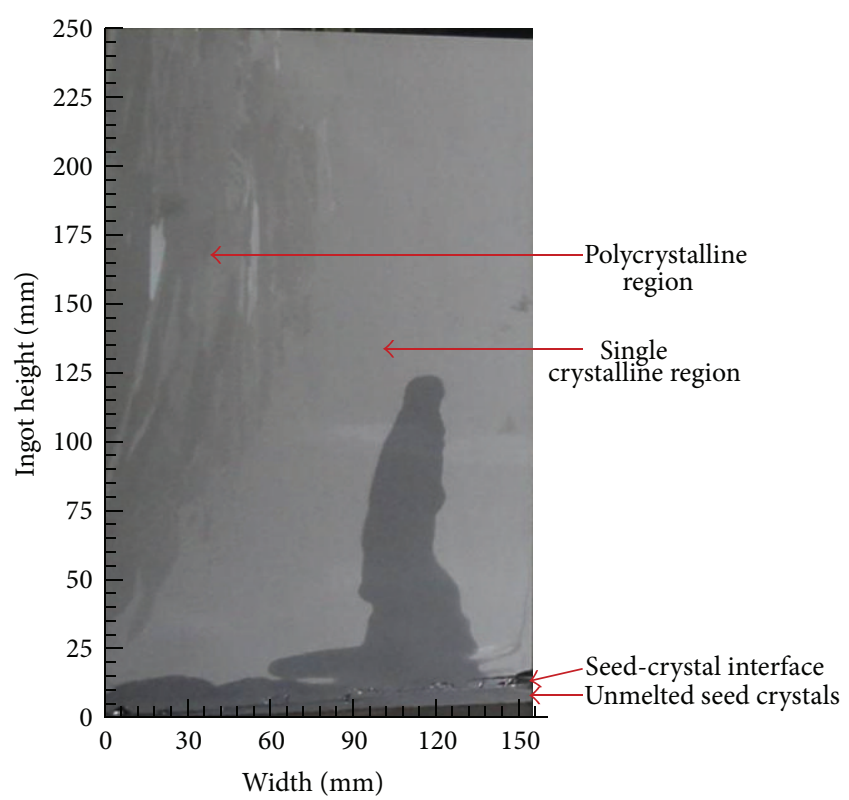

FIGURE 9: Preservation of seed crystals in the experiment. 
(no. 2010jdgz08), and the China Postdoctoral Science Foundation (no. 2013M540747).

\section{References}

[1] W. C. Ma, G. X. Zhong, L. Sun, Q. H. Yu, X. M. Huang, and L. J. Liu, "Influence of an insulation partition on a seeded directional solidification process for quasi-single crystalline silicon ingot for high-efficiency solar cells," Solar Energy Materials and Solar Cells, vol. 100, pp. 231-238, 2012.

[2] N. Stoddard, W. Bei, I. Witting et al., "Casting single crystal silicon: novel defect profiles from BP solar's mono ${ }^{2}$ wafers," Diffusion and Defect Data B, vol. 131-133, pp. 1-8, 2008.

[3] Q. H. Yu, L. J. Liu, W. C. Ma, G. X. Zhong, and X. M. Huang, "Local design of the hot-zone in an industrial seeded directional solidification furnace for quasi-single crystalline silicon ingots," Journal of Crystal Growth, vol. 358, pp. 5-11, 2012.

[4] A. Black, J. Medina, A. Piñeiro, and E. Dieguez, "Optimizing seeded casting of mono-like silicon crystals through numerical simulation," Journal of Crystal Growth, vol. 353, pp. 12-16, 2012.

[5] B. Gao, S. Nakano, and K. Kakimoto, "Influence of backdiffusion of iron impurity on lifetime distribution near the seedcrystal interface in seed cast-grown monocrystalline silicon by numerical modeling," Crystal Growth and Design, vol. 12, no. 1, pp. 522-525, 2012.

[6] X. Gu, X. G. Yu, K. K. Guo, L. Chen, D. Wang, and D. R. Yang, "Seed-assisted cast quasi-single crystalline silicon for photovoltaic application: towards high efficiency and low cost silicon solar cells," Solar Energy Materials and Solar Cells, vol. 101, pp. 95-101, 2012.

[7] N. Stoddard, R. Sidhu, J. Creager et al., "Evaluating BP solar's Mono $^{2}$ material: lifetime and cell electrical data," in Proceedings of the 34th IEEE Photovoltaic Specialists Conference (PVSC '09), pp. 1163-1168, Philadelphia, Pa, USA, June 2009.

[8] I. Witting, N. Stoddard, and G. Rozgonyi, "Defect incorporation and impurity precipitation in mono2 silicon," in Proceedings of the 18th Workshop on Crystalline Silicon Solar Cells and Modules: Materials and Processes,, pp. 155-158, Vail, Colo, USA, 2008.

[9] T. Tachibana, T. Sameshima, T. Kojima et al., "Evaluation of defects generation in crystalline silicon ingot grown by cast technique with seed crystal for solar cells," Journal of Applied Physics, vol. 111, no. 7, Article ID 074505, 2012.

[10] Y. Tsuchiya, H. Kusunoki, N. Miyazaki et al., "Correlation between carbon incorporation and defect formation in quasisingle crystalline silicon," in Procedings of the 38th IEEE Photovoltaic Specialists Conference (PVSC '12), pp. 297-301, Austin, Tex, USA, 2012.

[11] Z. Y. Li, L. J. Liu, X. Liu, Y. F. Zhang, and J. F. Xiong, "Heat transfer in an industrial directional solidification furnace with multi-heaters for silicon ingots," Journal of Crystal Growth, 2013.

[12] L. Liu and K. Kakimoto, "Partly three-dimensional global modeling of a silicon Czochralski furnace. I. Principles, formulation and implementation of the model," International Journal of Heat and Mass Transfer, vol. 48, no. 21-22, pp. 4481-4491, 2005.

[13] Z. Y. Li, L. J. Liu, X. H. Nan, and K. Kakimoto, "Role of marangoni tension effects on the melt convection in directional solidification process for multi-crystalline silicon ingots," Journal of Crystal Growth, vol. 346, no. 1, pp. 40-44, 2012.

[14] V. R. Voller and C. Prakash, "A fixed grid numerical modelling methodology for convection-diffusion mushy region phasechange problems," International Journal of Heat and Mass Transfer, vol. 30, no. 8, pp. 1709-1719, 1987. 

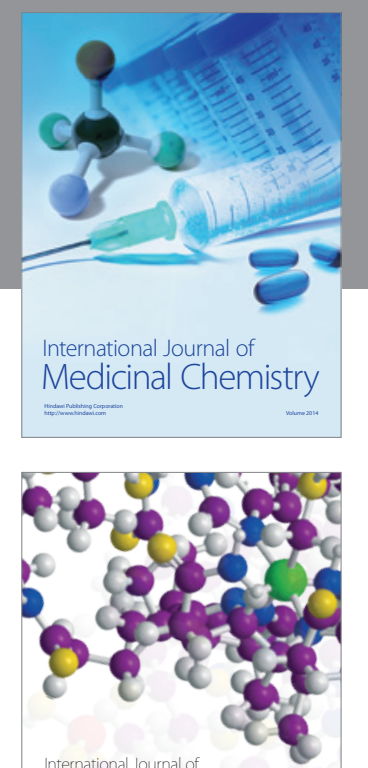

\section{Carbohydrate} Chemistry

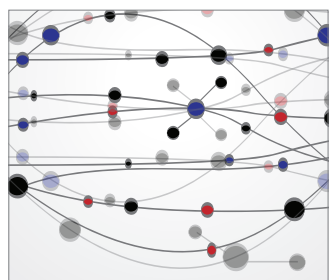

The Scientific World Journal
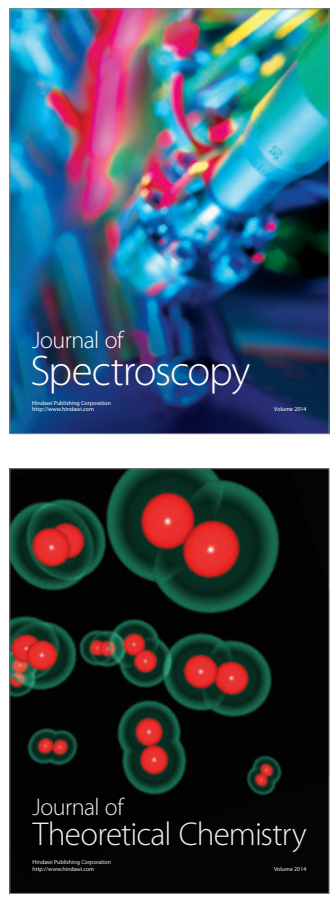
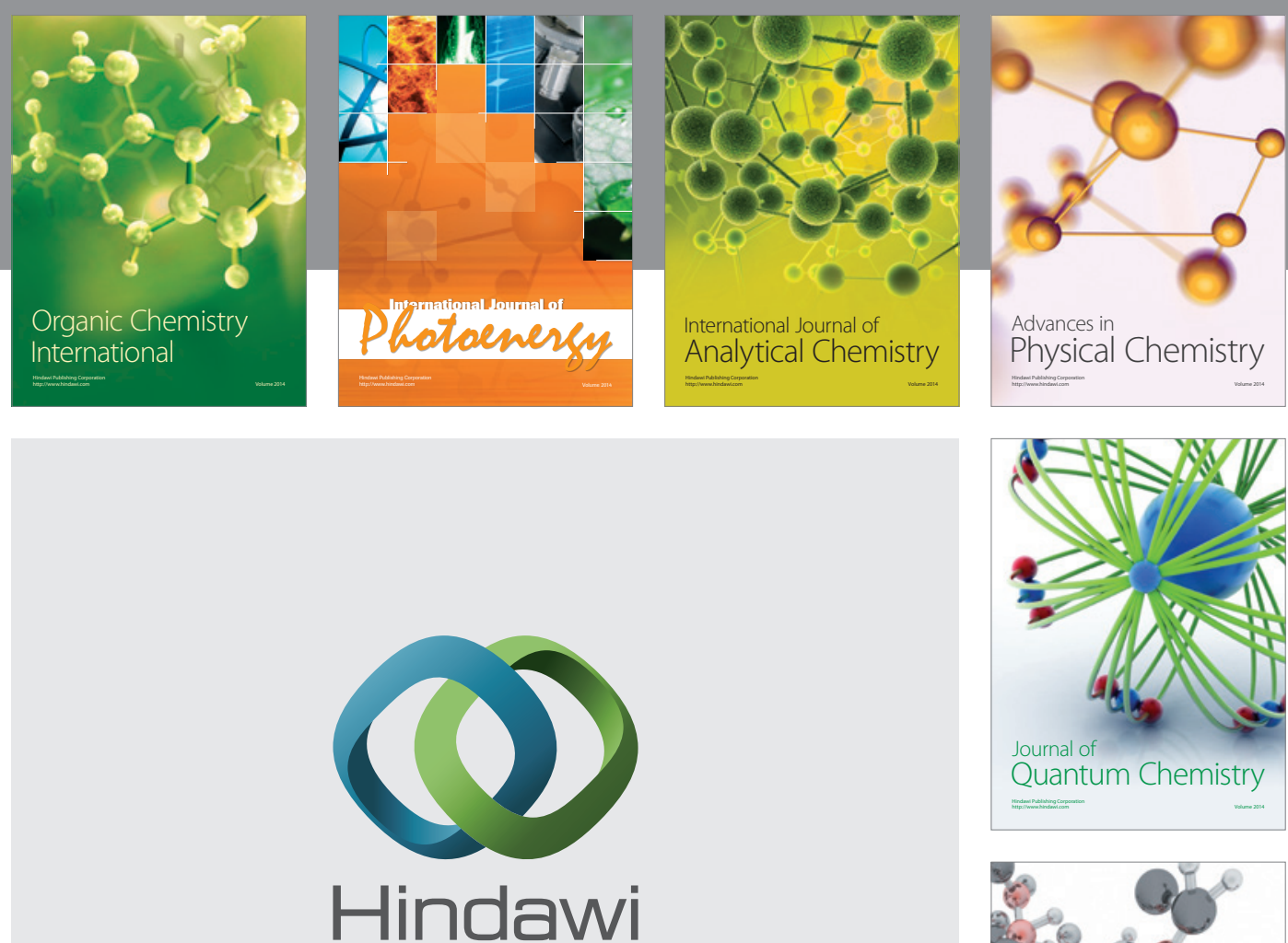

Submit your manuscripts at

http://www.hindawi.com

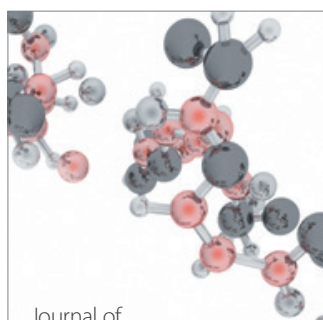

Analytical Methods

in Chemistry

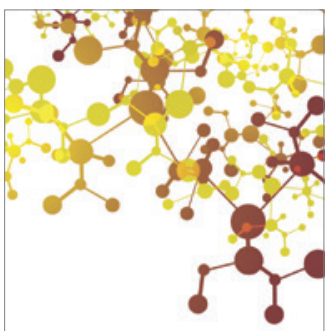

Journal of

Applied Chemistry

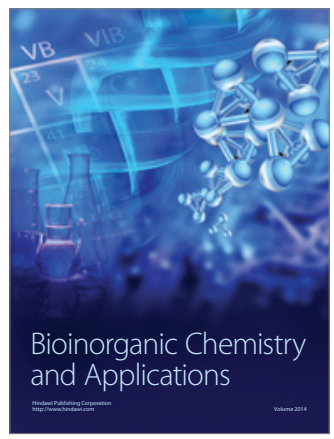

Inorganic Chemistry
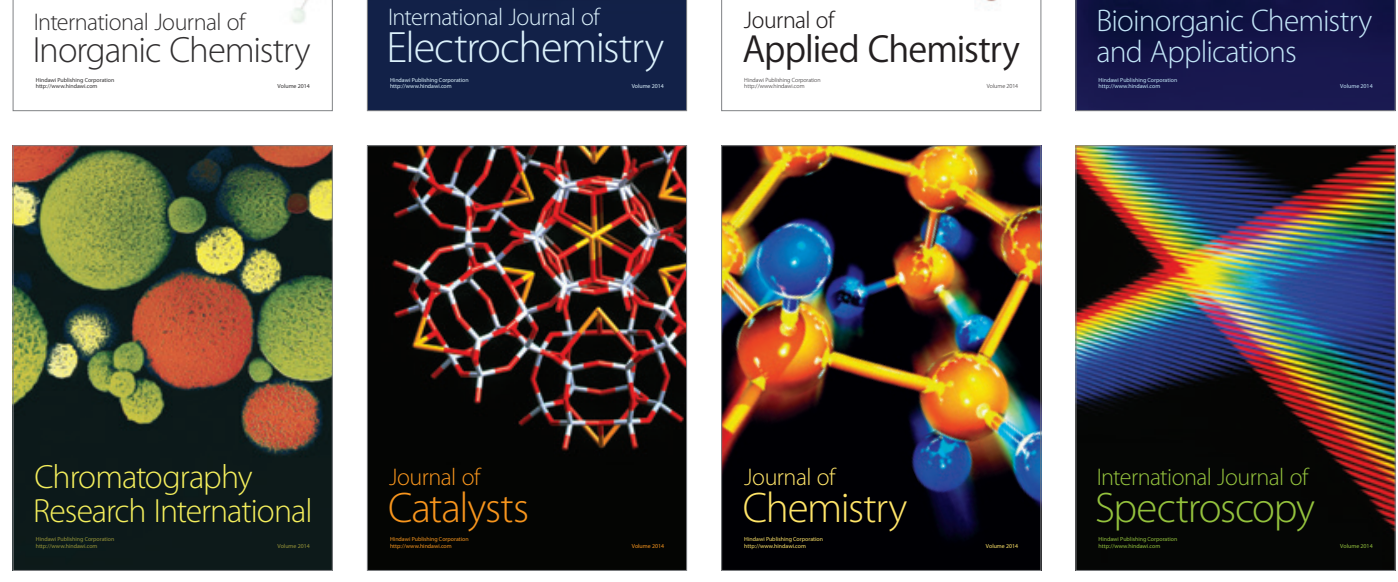